\title{
A Hybrid of Functional Networks and Support Vector Machine Models for the Prediction of Petroleum Reservoir Properties
}

\author{
Fatai Anifowose \\ Jane Labadin \\ Faculty of Computer Science and Information Technology, \\ Universiti Malaysia Sarawak, \\ 94300 Kota Samarahan, Sarawak, Malaysia \\ anifowo@kfupm.edu.sa_ljane@fit.unimas.my
}

\author{
Abdulazeez Abdulraheem \\ Department of Petroleum Engineering, \\ King Fahd University of Petroleum and Minerals, \\ Dhahran 31261, Saudi Arabia \\ aazeez@kfupm.edu.sa
}

\begin{abstract}
This paper presents an innovative hybrid of Functional Networks and Support Vector Machines (FN-SVM) as an improvement over an existing Functional Networks and Type-2 Fuzzy Logic (FN-T2FL) hybrid model. The former is more promising as it combines two existing techniques that are very close in performance and well known for their computational stability and fast processing. This proposed FNSVM hybrid model benefits from the excellent performance of the least-square-based model-selection algorithm of Functional Networks and the non-linear high-dimensional feature transformation capability that is based on structural risk minimization and Tikhonov regularization properties of SVM. Training and testing the SVM component of the hybrid model with the best and dimensionally-reduced variables from the input data resulted in better performance with higher correlation coefficients, lower root mean square errors and further less execution time than the standard SVM model. A comparison of FN-SVM with the existing FN-T2FL, using the same data and operating environment, showed that the FNSVM is more accurate and consumes less time.
\end{abstract}

Keywords-hybrid models, computational intelligence, porosity, permeability, functional networks, support vector machines.

\section{INTRODUCTION}

Computational Intelligence (CI) techniques such as Functional Networks (FN) and Support Vector Machines (SVM) have shown to be effective for a wide range of realworld applications. However, since each of them has its limitations and constraints that would not make it appropriate in solving all problems in different data and operational scenarios, there is the need for hybridization so that one technique would complement the limitation of others to ensure good performance in many challenging situations. Thus, hybridization of CI techniques can boost their individual performance and make them achieve much success in dealing with large-scale, complex problems.

The application of CI techniques and their hybrids is especially appreciated in the oil and gas exploration and production since a little improvement in accuracy of the prediction of various petroleum reservoir properties could lead to a very high increase in the discovery and production of more energy. The two important properties of oil and gas reservoirs that are focused in this study are porosity and permeability. These are the fundamental reservoir properties that relate to the amount of fluid contained in a reservoir and its ability to flow. They are frequently measured in the laboratory on plugs extracted from the core of wells drilled for oil and gas exploration and serve as standard indicators of reservoir quality in the oil and gas industry.

Since the laboratory measurements are usually costly and time-consuming, Artificial Intelligence (AI) techniques [1-4] as well as hybrid methodologies [5-8] have been successfully applied in the prediction of these properties to a reasonable degree of accuracy. However, each AI technique has certain limitations that would not make its application desirable in certain conditions such as small dataset scenarios $[9,10]$ and high dimensionality of data conditions $[11,12]$. Since FN uses a least-square algorithm that selects the best subset of features from a set of input data and SVM is known for its capability to conveniently handle data of high dimensionality with its insensitivity to data size, combining these two techniques in a way that they complement each other would be a welcomed development. Such hybridization task would improve the accuracy of predictions, which would in turn, increase the confidence in the results that would improve the efficiency of exploration and production activities.

This study presents a prudent combination of $\mathrm{FN}$ and SVM methodologies to build a hybrid intelligent model that outperforms the results produced by an existing FN and Type-2 Fuzzy Logic (FN-T2FL) hybrid model [13]. The desirable qualities of FN and SVM were combined to predict the two properties of oil and gas reservoirs with better performance indices. Our major motivation for this study is the continued discovery of various CI techniques with common denominators that are suitable for hybridization.

\section{SURVEY OF Literature}

\section{A. Oil and Gas Reservoir Characterization}

Oil and gas reservoir characterization is a process for quantitatively describing various reservoir properties in spatial variability using available field and laboratory data [1]. Reservoir characterization plays a crucial role in modern reservoir management. It helps to make sound reservoir decisions and improves the asset value of the oil and gas companies. It maximizes integration of multidisciplinary data and knowledge, and hence improves the reliability of reservoir predictions. The ultimate goal is a reservoir model with realistic tolerance for imprecision and uncertainty [6]. Reservoir characterization focuses on modeling each 
reservoir unit, predicting well behavior, understanding past reservoir performance, and forecasting future reservoir conditions.

The measurement of various reservoir properties is made on plugs of rock samples. Others are made on devices inserted into oil wells on site. These measurements are called logs and the process of taking the log measurements is called well logging [14]. Different borehole and reservoir formation conditions may require different tools to measure the same property. There are many important subsurface properties that need to be detected or measured but porosity and permeability are the most important since they both serve as major indicators of petroleum reservoir quality and economic viability. The data acquired from these datasets are used for the estimation of porosity, permeability and other reservoir properties such as rock types, the thickness of rock layers; the amount of hydrocarbons; and water salinity.

Porosity is an important consideration when attempting to evaluate the potential volume of hydrocarbons contained in a reservoir as it is a measure of the percentage of voids and open spaces in a rock. These voids and spaces are potential receptacles for oil and gas. Permeability, on the other hand, is a key parameter in the characterization of any hydrocarbon reservoir as it is a measure of how interconnected the individual voids and spaces are in a rock. In fact, many petroleum engineering problems cannot be solved accurately without having an accurate value of permeability $[15,16]$. In view of the importance of porosity and permeability in oil and gas exploration and production, this study focuses only on these two properties.

\section{B. Hybrid Computational Intelligence in Oil and Gas}

Hybrid Computational Intelligence (HCI) has been generally and widely applied in the bioinformatics, science, technology and engineering. Such applications include the selection of winding material in electric power transformers using a hybrid of decision trees for attribute selection and neural networks for winding material classification for the calculation of the performance characteristics of each considered design [17]. Reference [18] used a hybrid of genetic programming approach and a heuristic rule-based scheme for the classification between different types of aphasia, a human syndrome, often due to brain damage. Other advances of $\mathrm{HCI}$, especially in oil and gas, include $[19,20,21]$.

In petroleum engineering, HCI has been successfully used in many areas of application such as seismic pattern recognition; porosity and permeability predictions; identification of sandstone lithofacies; drill bit diagnosis; and analysis and improvement of oil and gas well production. Reference [22] used a hybrid of Fuzzy Logic and Artificial Neural Networks (ANN) for the prediction of permeability by means of Flow Zone Index. A fusion of Genetic Algorithm (GA) and ANN was used to estimate reservoir permeability by [23] while using the GA to automatically tune the parameters and ANN to establish a relationship between the log data and core permeability. A hybrid FuzzyGA system for the optimization of gas production operations was proposed by [24]. They used the traditional Fuzzy Logic to accommodate uncertainties in the field data and GA as a primary optimization scheme to solve the optimum gas production rates of each well and pipeline segment diameters to minimize investment costs. Other related works on the application of HCI in oil and gas include [25-28].

Most of the previous studies featured GA, Fuzzy Logic and ANN in their proposed hybrid models. Though, GA is a very robust optimization algorithm that is based on an exhaustive search paradigm, it is well known for its long execution time, its need for high processing power due to its computational complexity and sometimes inefficiency [29]. Fuzzy Logic becomes complex and time-consuming when applied on high-dimensional data [12] and performs poorly when applied on datasets of small size [13]. ANN is also known to suffer from many deficiencies such as having no general framework for the design of its appropriate network for a specific task and its frequent requirement of large number of parameters to fit a good network structure [30].

A closely related work is [6]. However, the authors used three-component hybrid models comprising of FN, T2FL and SVM, making the models complex to analyze. In addition, there seems to be a redundancy in the role of the T2FL and SVM components in the hybrid models. Hence, this paper focuses on a simple design and clear role assignment and expectations to its two hybrid components.

FN and SVM have been reported to possess excellent least-square fitting capabilities and efficient scalability with both small and large datasets $[6,13,31]$. This accounts for their choice for hybridization in this study.

\section{DESCRIPTION OF DATA, EXPERIMENTAL DESIGN AND MODEL FRAMEWORK}

\section{A. Description of Data}

The same sets of porosity and permeability data from six wells (three for porosity and three for permeability) from previous studies $[6,7,13]$ were used in this work. Site 1 , a heterogeneous platform that is made up of carbonate and dolomite, contains six predictor variables for porosity while site 2, majorly of carbonate and sandstone formations, contains eight predictor variables for permeability. Hence, the datasets are representative of the major oil-bearing geological structures found in most parts of the oil-producing world.

\section{B. Experimental Design}

The methodology employed in this study follows the standard computational intelligence approach to hybridization of AI techniques, and in the case of this study, a combination of FN and SVM techniques. The MATLAB codes for the iterative least-squares FN classifier obtained from the software repository of Enrique Castillo's AI Research Group [32] were partly used while the Least Squares-SVM (LS-SVM) whose basic version is available at [33] were used for SVM. Both were then customized with various toolboxes and functions. FN and SVM were purposely chosen as preferred candidates for hybridization due to their common features in terms of good performance 